Irish Section Conference, 22-24 June 2021, Nutrition, health and ageing — translating science into practice - Part A

\title{
Profiling children's snack products in Ireland
}

\author{
S. Mooney ${ }^{1}$ and E. L. Feeney ${ }^{2}$ \\ ${ }^{1}$ School of Agriculture and Food Science, University College Dublin, Belfield, Dublin, Ireland and \\ ${ }^{2} U C D$ Institute of Food and Health, University College Dublin, Belfield, Dublin, Ireland
}

Infancy and childhood are critical periods for growth and development, requiring adequate nutrition. Early feeding experiences can have a lasting impact on food preferences and life-long eating habits ${ }^{(1)}$. The high rate of childhood obesity, along with the establishment of new healthy eating guidelines in Ireland, demonstrates the importance of developing best practice in infant feeding. While 'healthy' snacks may be offered 2-3 times a day, a wide range of commercial snack products, varying in nutrient content and textures, now exists. Many of these are known to be marketed inappropriately, in other European countries ${ }^{(2)}$, due to their age recommendations and/ or their nutrient content. The aim of this study was to analyse the available infant snack foods on the Irish market and examine their energy and nutrient content, recommended ages, and potential messages around marketing.

A database of commercially-available infant snack products in Ireland was created, focusing on those recommended for ages 4-12 months. Information collected included the name, brand, price, packaging, nutrient content per $100 \mathrm{~g}$ and per recommended portion, ingredients, recommended age and any nutrition/health claims. $\mathrm{n}=148$ products were identified, which fell into nine categories of snack type. Statistical analyses was conducted using SPSS v26 for Mac (IBM). One-way Analysis of Variance (ANOVA) was used to compare nutrient content across categories.

Nine snack categories were identified ('Extruded snacks', 'Fruit-based pouches', 'Fruit-based snacks', 'Biscuits', 'Rice cakes', 'Fruit \&veg snacks', 'Bars', 'Wafers', and 'Houmous snacks'. Snack products overall were relatively energy-dense, nutrient-poor and contributed only limited micronutrients. The majority contained relatively high levels of total sugars and fat as defined by the $\mathrm{WHO}^{(3)}$; with almost one-third of products $(n=44)$ containing $30 \%$ or more energy from total sugars, and $n=94$ products containing over $4.5 \mathrm{~g}$ of fat per $100 \mathrm{~g}$. 'Houmous' products and 'Extruded snacks' contained the greatest energy from fat, at $47 \%$ and $29.2 \%$ energy, on average, respectively, followed by 'bars' (26.7\% energy) and 'biscuits' (23.9\% energy). Fruit-based pouches and fruit-based snacks contained the greatest energy from total sugars, at $75.7 \%$ and $69.7 \%$, respectively. Regarding age recommendations, $12.2 \%$ of products were advertised as suitable from 4 months of age.

A high proportion of the available snack food products targeted at young children aged 4-12 months contained high levels of energy from fat, and from total sugar, in this supermarket analysis. Of the 148 products in this study, 12.2\% were advertised as suitable for infants from 4 months of age, which contradicts WHO recommendations that no foods should be recommended to children before 6 months of age.

\section{References}

1. Beauchamp, GK \& Mennella JA (2011) Digestion 83, Suppl 1, 1-6

2. World Health Organization (2019) Improving the nutritional quality of commercial foods for infants and young children in the WHO European region. [Available at: https://www.euro.who.int/_data/assets/pdf_file/0008/407564/Improving-Nutritional-Quality-LowRes.pdf]

3. AE Bennett, O’Connor A, Canning N, et al. (2012) Ir Med J 105 